Genij Ortopedii. 2022. Vol. 28, no. 1. P. 104-110.

Original article

https://doi.org/10.18019/1028-4427-2022-28-1-104-110

\title{
Opposable triphalangeal digital stump transplanted with microvascular anastomoses in patients with frostbitten hands and feet (case report)
}

\author{
Nikolai M. Aleksandrov ${ }^{\bowtie}$, Ivan D. Veshaev, Dmitry A. Kuptsov
}

Privolzhsky Research Medical University, Nizhny Novgorod, Russian Federation

Corresponding author: Nikolai Mikhailovich Aleksandrov, aleksandrov-chetai@rambler.ru

\section{Abstract}

The objective was to explore possibilities with reconstruction of opposable digital stump of the hand transplanted with microvascular anastomoses in frostbite patients. Material and methods Medical histories and long-term results of treatment of two frostbite patients with finger stumps of both hands were reviewed. The triphalangeal finger and the thumb were reconstructed by transplanting the opposable finger stump together with the metacarpophalangeal joint using microvascular and microneural anastomoses. The stumps were mobilized and transplanted to the distal ulnar artery with the venous drainage performed using the dorsal saphenous vein of the hand. Long-term results were examined at 10 years and 2.5 years of surgery. Clinical, radiological, biomechanical and biophysical investigations were produced. Results The bilateral gripping function was restored in recipient stumps without functional damage to the donor stumps. Discriminatory sensitivity of reconstructed fingers was $6 \mathrm{~mm}$ and $8 \mathrm{~mm}$, the range of active motion in the transplanted joint was 55 and 66 degrees. Discussion Opposable digital stump transplantation with microvascular anastomoses has significant advantages over the classical surgery. The technique allows for one-stage restoration of a finger to have sufficient length, function, adequate blood supply and innervation due to a short digital stump being transplanted with the metacarpophalangeal joint. The advantage over a toe transplant consists of the use of digital structures of the hand that are identical in functionality and the anatomy reducing the need for secondary interventions. Conclusion The method of management can be successfully applied for frostbite patients with hand stumps according to indications.

Keywords: finger stump transplant, finger reconstruction, frostbite, microvascular anastomosis

For citation: Aleksandrov N.M., Veshaev I.D., Kuptsov D.A. Opposable triphalangeal digital stump transplanted with microvascular anastomoses in patients with frostbitten hands and feet (case report). Genij Ortopedii, 2022, vol. 28, no 1, pp. 104-110. https://doi. org/10.18019/1028-4427-2022-28-1-104-110

\section{INTRODUCTION}

Toe transplantation by microvascular anastomosis is one of the major methods of finger reconstruction used for congenital pathology and consequences of mechanical trauma [1-6]. Surgeons' attitudes toward the interventions performed for cold-induced injury are more restrained because of a high risk of thrombosis of anastomosis and graft collapse due to cold endarteritis of the upper and lower limbs [7]. Patients who have suffered frostbite often have stumps of the toes and feet that significantly limit the potential of the methoda and relevant donor resources of the contralateral hand are essential. In comparison with the transplantation of the toe or its segments, the transplantation of tissue complexes of the contralateral hand for finger reconstruction is occasionally used for mechanical and cold-induced injuries [8]. Transplantation of a full length finger of the contralateral hand is rare in frostbite due to the finger stumps in both hands, and one of a finger stump can be transplanted. However, possibilities with the method, technical and strategical approaches to the interventions depending on the functional state of the vessels, a defect type and the level of finger and hand stumps have been underexplored. Few cases with non-cold injuries treated with the method of digital transplantation are reported without description of the surgical technique [9-11].

The objective was to explore possibilities with reconstruction of opposable digital stump of the hand transplanted with microvascular anastomoses in frostbite patients.

\section{MATERIAL AND METHODS}

The study was performed in accordance with ethical principles for medical research involving human subjects stated in the Declaration of Helsinki (1975) developed by the World Medical Association as revised in 2008. Clinical, radiological, biomechanical and biophysical methods were employed in the study. Transplantation of a finger stump of the contralateral hand was performed in two male patients with stumps of both hands after frostbite.

Clinical cases of reconstruction of the threephalangeal finger and the thumb by transplanting a complex of tissues of the contralateral hand by microvascular anastomoses are reported. A 31-year-old patient $\mathrm{K}$. was treated at the hospital for the stumps of

○ Aleksandrov N.M., Veshaev I.D., Kuptsov D.A., 2022 
the three-phalangeal fingers of the left hand at the level of the heads, the stump of the fourth finger at the level of the distal third of the main phalanx, the fifth finger at the level of the middle third of the main phalanx of the right hand, bilateral stump of the first toe at the level of the heads of the main phalanges after frostbite that occured two years ago (Fig. 1 a, b, c). On admission, the stumps of the second, third, fourth and fifth metacarpals of the left hand were identified at the level of the heads covered with scars that fused with the bone.

The ends of the stumps of the fourth, fifth fingers of the right hand had adequate skin appearance with discriminatory sensitivity of $4-5 \mathrm{~mm}$, full ROM in the metacarpophalangeal joints. The lateral grip of the left hand was sharply limited and there was no pinch grip. The patient was offered second and third toe transplantation by microvascular anastomoses to the position of the third and fourth fingers of the left hand. The patient refused the intervention and was offered transplantation of the stump of the fourth finger of the right hand to the position of the third finger of the left hand. The longer donor stump was an appropriate choice to save the function of the hypotenar muscles involved in the pinch grip and opposition of the thumb that would have been sacrified with transplantation of the stump of the fifth finger and the metacarpophalangeal joint. In addition to that, the appearance of the hand would have been deteriorated with involved contour of the radial aspect of the hand. The Allen test showed confinement of the palmar arterial arches. A wave-shaped skin incision was made on the palmar surface of the right forearm extended to the palm, and a U-shaped skin-fat flap with a triangular apex and a distal base was harvested in the projection of the fourth metacarpal. The ulnar artery was mobilized together with the superficial palmar arch and the common finger arteries of the third and fourth interdigital spaces. The palmar arch was ligated at the level of the third metacarpal bone. Then the palmar finger arteries extending to the stump of the fifth and the third finger were ligated. The common finger nerves in the area of the third and fourth interdigital spaces were split to the level of the proximal third of the palm. To do this, the epineurium was dissected on the palmar surface of the common finger nerve along the fibers from the division site to the proximal third of the metacarpals. The nerve bundles forming the finger nerves were separated from each other from the level of division in the proximal direction within the epineurotomy performed in a blunt way. Innervation of the donor stump and the adjacent fingers and stumps was preserved. The stump of the fourth finger included two common and palmar finger arteries, nerves and flexor tendons covered with a skin-fat flap. An identical incision was also made on the dorsal aspect of the hand with a similar U-shaped skin-fat flap harvested in the projection of the third metacarpal. The largest dorsal subcutaneous vein was mobilized with a single collector formed to the level of the lower third of the forearm and the dorsal subcutaneous veins of the stump of the fourth finger preserved. A transverse osteotomy of the fourth metacarpal was performed at the level of the middle third with the dorsal and palmar interosseous muscles cut off and the intermetacarpal ligaments intersected. The stump of the finger and the metacarpophalangeal joint were mobilized on the ulnar artery and the dorsal subcutaneous vein, the tendons of the flexors and extensors of the fourth finger and the finger nerves. The end of the stump of the third metacarpal of the left hand was exposed, scars excised, the wound extended to the palm and the lower third of the forearm, and the finger nerves, radial artery and cephalic vein were isolated. The nutrient vessels were intersected at the level of the lower third of the forearm, and the nerves and tendons of the flexors and extensors of the stump of the fourth finger transected at the level of the proximal third of the hand, and the stump of the fourth finger of the right hand was transplanted together with a fragment of the metacarpal to the position of the third finger of the left hand. Osteosynthesis of the metacarpal fragment, the graft and the stump of the third metacarpal was performed after their ends were processed with cylindrical cutters and additionally fixed with Kirschner wire (Fig. 1 d). The finger nerves were sutured with 9/0 material. Microvascular anastomoses were applied end-to-end between the graft artery and the radial artery, the mobilized dorsal subcutaneous vein of the hand with the cephalic vein using 9/0 suture material and an Opton surgical microscope. The tendons of the flexors and extensors of the stump of the fourth finger and the tendons of the third finger were sutured at the level of the middle third of the metacarpals. Defects on the lateral aspects of the transplanted fragment of the fourth metacarpal were left open for delayed closure with skin grafts. The donor wound was sutured primarily on the palmar and dorsal aspects of the hand and the forearm after approximation of the third and the fifth metacarpals. Fluid and anticoagulant therapy was performed for 7 days postoperatively. The postoperative period was uneventful, the blood supply to the transplanted stump remained adequate. The transplanted tissue complex and the skin grafts completely survived. Wounds in the donor and recipient areas healed by primary intention. There were no signs of vascular insufficiency of the preserved fingers and their stumps on the donor and recipient hands (Fig. $1 \mathrm{e}, \mathrm{f}, \mathrm{g}, \mathrm{h}$ ). 


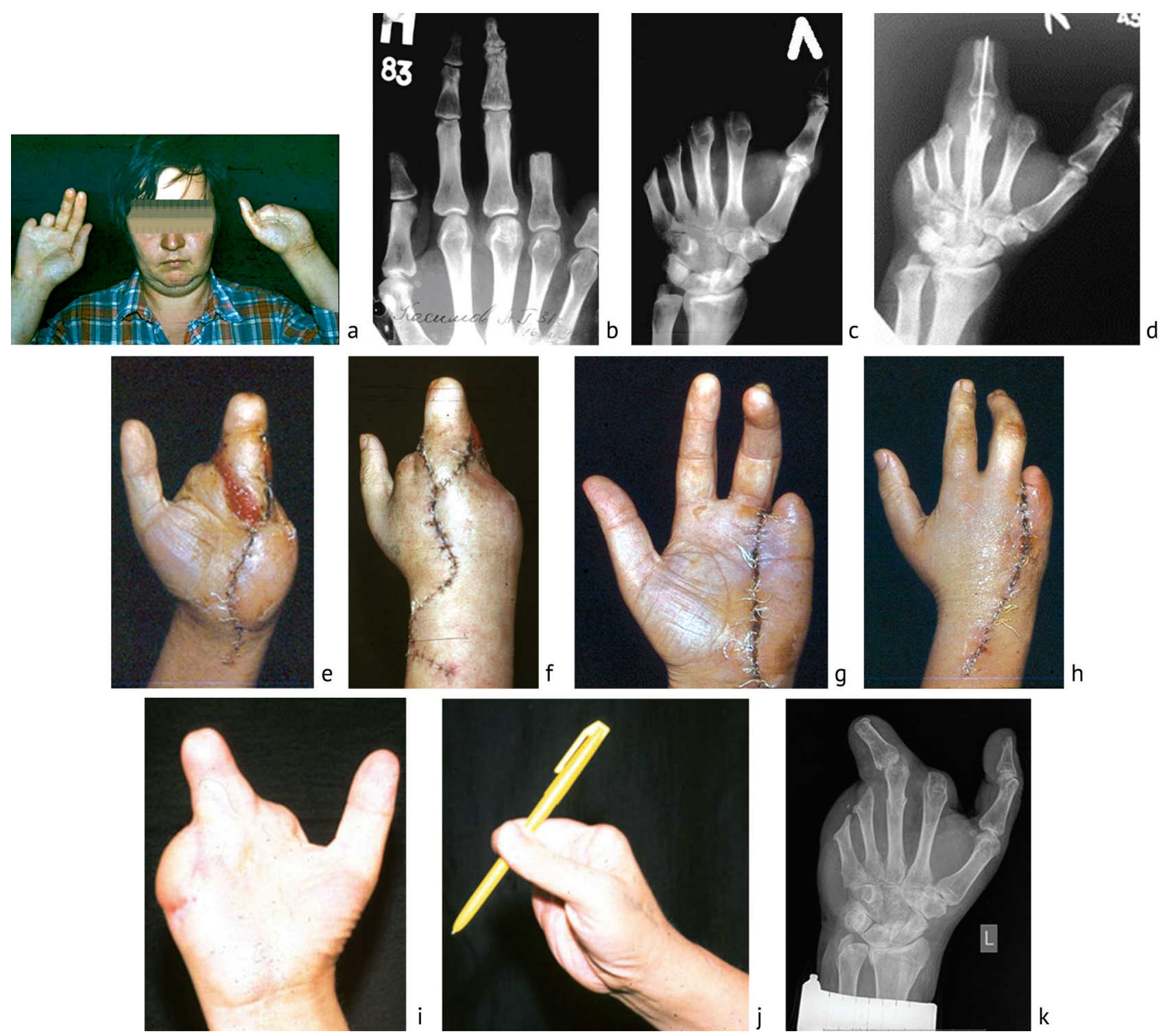

Fig. 1 The result of treatment of a 31-year-old patient K. with finger stumps of both hands: (a) preoperative appearance of the hands; (b) preoperative radiograph of the donor (right) hand; (c) preoperative radiograph of the recipient (left) hand; (d) radiographs of the hands two weeks after surgery; (e) appearance of the left hand two weeks after finger stump transplantation (palmar aspect); (f) appearance of the left hand two weeks after finger stump transplantation (dorsal aspect); (g) appearance of the donor hand two weeks after surgery (palmar aspect); (h) appearance of the donor hand two weeks after surgery (palmar aspect); (i) appearance of the donor hand two weeks after surgery after surgery (dorsal aspect); (j) appearance of the hand and extension of the stump of the fourth finger of the right hand transplanted to the stump of the third metacarpal of the left hand; (k) pinch grip of the left hand; (l) radiograph of the left hand at a long term

A 37-year-old patient K. was treated at the hospital for the stumps of both hands and the first toe of the right foot after frostbite that occurred about 2 years ago. He had stumps of the first and second metacarpals at the level of the distal third, stumps of the third, fourth, fifth metacarpals at the level of the proximal third of the right hand (Fig. 2 a). The movements of the stump of the first metacarpal bone were preserved with no grip of the hand due to the absence of the ulnar edge of the palm. He had stumps of the second finger at the level of the base of the middle phalanx, the stump of the third finger at the level of the middle third of the main phalanx, the fourth finger at the level of the distal third, the fifth finger at the level of the middle third of the metacarpal bone of the left hand. The movements of the stumps in the metacarpophalangeal joints were preserved. There was a rough, stiff scar, tightly fused with the stumps and painful on palpation at the ends of the stumps of the second, third, fourth, fifth metacarpals on the right. Similar scars were present on the stumps of the fourth and fifth metacarpals of the left hand. The patient underwent reconstruction of the ulnar edge of the right hand earlier. The first stage of treatment was performed to excise the scars on the ends of the stumps of the second, third, fourth, fifth metacarpal bones with the replacement of the resultant tissue defect using a non-free skin-fat inguinal flap. Soft tissues of the ulnar edge of the hand were formed by duplication of the skin-fat flap (Fig. 2 b). The inguinal flap survived completely with the donor and recipient wounds healed by primary intention. Two months later prior to the next 
stage of treatment the patient showed adequate, elastic skin that covered the ends of the stumps of the second, third, fourth, fifth metacarpal bones and an excessive, movable flap in the area of the ulnar edge of the stump formed by duplication. The second stage included bone reconstruction of the ulnar edge of the hand by transplanting a fragment of the second metatarsal bone of the left foot with a sentinel skin-fat flap by microvascular anastomoses in combination with interposition plasty using a vascular free cortical-cancellous graft harvested from the iliac wing to the stump of the fourth metacarpal. Duplication leaflets were separated from a skin-fat flap. The grafts were located between the duplication leaflets. The blood supply to the graft was restored by anastomosing the artery of the nutrient pedicle, represented by the dorsal artery of the foot, the ulnar artery, the medial subcutaneous vein of the foot, incorporated in the graft, with the dorsal subcutaneous vein of the forearm. Osteosynthesis was performed by inserting the ends of bone fragments after processing with cylindrical cutters and additional fixation with wires. Bone grafts were covered with soft tissues formed at the previous stage, the sentinel flap was placed on the palmar surface of the formed ulnar counteract branch (Fig. 2 c, d). The blood supply remained adequate and indicated the preserved blood supply to the transplanted fragment of the second metatarsal. Skin flaps and bone grafts survived completely. Reconstruction of the first finger of the right hand was performed a year later. An 8 $\mathrm{cm}$ ulnar branch covered with a sufficient amount of soft tissues was identified on admission. No transplantation was performed due to the presence of the stump of the first toe of the left foot and a post-resection defect of the second metatarsal to avoid a defect in the other foot. Transplantation of one of the finger stumps of the opposite hand was produced for reconstruction of the thumb of the right hand. The conducted Allen test showed confinement of the palmar arterial arches. the shortest stump of the third finger was transplanted to minimize the donor defect of the hand (Fig. 2 e, f). The technique of mobilization, transplantation of the stump and restoration of blood flow were similar to those described in the first case.
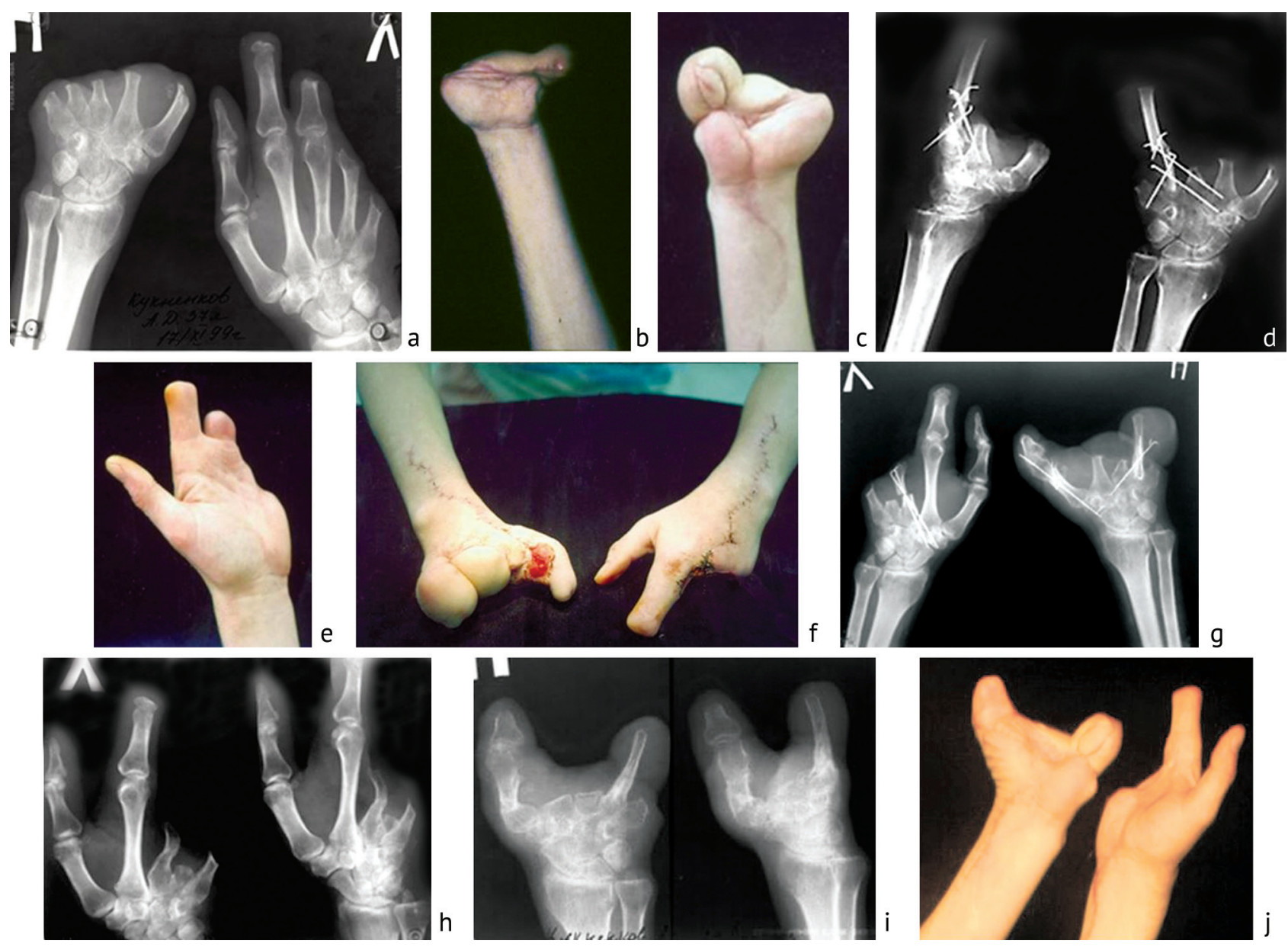

Fig. 2 The result of treatment of a 37-year-old patient K. with finger stumps of both hands: (a) preoperative radiographs of both hands; (b) appearance of the right hand after replacement of scars and the soft tissues formed at the ulnar edge of the hand using an inguinal skin-fat flap; (c) appearance of the right hand with the bone base formed, the sentinel flap of the blood-supplied skin-bone fragment is visible; (d) radiographs of the right hand showing the bone base formed at the ulnar edge of the hand; (e) appearance of the donor hand; (f) appearance of the donor and recipient stump of the hand two weeks after transplantation of the stump of the third finger; (g) radiographs of the hands two weeks after transplantation of the stump of the finger on the right hand and the transposition of the stump of the fourth metacarpal on the left hand; (h) radiographs of the left hand 2.5 years after surgery; (i) radiographs of the right hand 2.5 years after surgery; (j) appearance of both hands 2.5 years after surgery 
Osteosynthesis of the metacarpal fragment of the transplanted stump and the stump of the first metacarpal was performed with the insertion technique after treatment with cylindrical cutters and fixation with a wire (Fig. $2 \mathrm{~g}$ ). The wound on the medial surface of the transplanted stump was left open for delayed closure with skin grafts. The stump of the fourth metacarpal bone was transferred to the position of the third metacarpal bone with metacarpal fragments fixed with a wire to approximate the edges of the donor wound and ensure the possibility for primary suturing (Fig. 2g). The transplanted stump and the split skin grafts survived completely. The blood supply to the preserved fingers and stumps of the donor and recipient hands remained adequate. Donor and recipient wounds healed by primary intention.

\section{RESULTS}

The first patient was examined 10 years after the operation (Fig. 1g, h, i). The function of the opposition of the first to the stump of the third finger, the lateral grip of the left hand, pain and temperature sensitivity of the third finger reconstructed were restored. The discriminatory sensitivity of the transplanted stump was $8 \mathrm{~mm}$. The range of active movements in the metacarpophalangeal joint of the finger was 66 degrees. The distance between the distal part of the transplanted stump and the first finger at abduction was $11 \mathrm{~cm}$. There were no radiological signs of resorption at the distal parts of the stump. The articular space of the transplanted joint could be clearly seen, with the contours of the articular ends being well-defined and having a normal structure. The function of the stump of the fifth, the second and the third fingers of the donor hand was intact. The patient reported no changes in the appearance of the donor hand after the operation. The patient could use both hands in daily activities.

The second patient was examined 2.5 years after the operation (Fig. 2h, i, j). The length of the restored finger with the metacarpal bone was $9 \mathrm{~cm}$, and the ulnar branch formed was $8 \mathrm{~cm}$, as per postoperative measurements. The first finger was functionally positioned. The range of active movements in the transplanted metacarpophalangeal joint of the stump was 55 degrees. The double-sided grip of the hand was restored. The distance between the ends of the ulnar branch and the restored first finger at the maximum abduction was $10 \mathrm{~cm}$. The pain and temperature sensitivity of the first finger was identified. The donor defect on the left foot was insignificant and of no concern for the patient. The control radiographs of the right hand showed complete consolidation of the third metacarpal fragment transplanted with the finger stump, complete consolidation and reconstruction of the avascular graft and the second metatarsal fragment. The metacarpophalangeal joint of the first finger had normal structure, the articular space, the contours of the articular ends were clearly visualized. There were no signs of resorption of the transplanted stump. There were no restrictions in the function of the donor hand and the patient could use both hands in daily activities. The patient did not report deterioration in the appearance of the donor hand. Radiographs of the donor hand showed complete consolidation of the stump of the fourth metacarpal transplanted to the proximal part of the third metacarpal bone (Fig. 2h, i).

\section{DISCUSSION}

Both classical and precision techniques can be used to transplant the finger of the opposite hand. The transplantation of the finger of the opposite hand in the classical version consists in the use of a temporary pedicle flap containing finger neurovascular bundles as described in the monograph by B.V. Parin [12]. The advantage of the method is the possibility of using it regardless of endarteritis, injury to the finger vessels and absence of the likelihood of vascular thrombosis. Our clinic has been experienced in transplanting a finger of the opposite hand on a temporary pedicle in 3 patients with consequences of mechanical trauma. Osteosynthesis of the transplanted segment and the stump of the restored finger was produced at the first stage, the donor and recipient hands were fixed to each other for 4 weeks before vascular links formed in the recipient bed and the donor finger with the onset of the peripheral revascularization. The length of the pedicle needed was provided by resection of the main phalanx of the donor finger, and the method could not be used for metacarpal transplantation with or without the metacarpophalangeal joint because of unavailable length required for the transplanted segment of the hand and the adequate length of the restored finger. For this reason, the method was used to reconstruct the thumb stump at distal levels with the mobility provided by the tenar muscles. The pedicle intersected from the donor hand at the second stage resulted in impaired integrity of the neurovascular bundles, sensitivity and major circulation in the restored finger. There was an impaired venous outflow and restricted ROM in the interphalangeal joints with restored flexor and extensor tendons. For this reason, B.V. Parin recommended transplanting a defective 
finger only for the reconstruction of the thumb maintaining the sensitivity of the remaining threephalanges and never transplanted an intact finger. The disadvantages of classical methods are twostage, prolonged forced position of the limbs, sharply restricted ROM in the joints, lack of sensitivity of the restored finger, the possibility of transplanting only the nail and middle phalanges of the preserved threephalanx finger. In our opinion, the method can be used only in single cases with injury to the finger vessels and pre-distraction of the pedicle with a transosseous apparatus to provide the possibility of transferring a segment of the required length.

The results of finger stump transplantation by microvascular anastomoses show that the use of microsurgical technique allows to restore fingers of sufficient length with adequate blood circulation and innervation at one stage that is in line with the results of transplantation performed for the consequences of mechanical trauma [10]. Sufficient blood supply to the tissues of the transplanted stump also ensures adequate function of the joints and tendons. The technique facilitates full range of movements in the joint of the transplanted stump and the possibility of restoring the pinch grip and opposition of the thumb, even in incomplete restoration of the finger anatomy. Compared with a toe transplant, the advantage of the method is the use of tissues and anatomical formations structurally identical to donor ones. From a biomechanical point of view, the metacarpophalangeal joint in contrast to the metatarsal-phalangeal joint provides more flexion with baseline deficient flexion and predominant extension in the metatarsal-phalangeal joint. There is no need to perform corrective interventions for the joints of the restored finger, as is the case with a toe transplant $[5,14-17]$. In addition to that, a vascular pedicle of the required length with a sufficient diameter of the vessels can be formed to avoid autovenous plasty and reduce the risk of thrombosis of microanastomoses.
The persistent vascular anatomy, a large diameter of the anastomosed vessels allows for a stump transplantation with high reliability.

Our small clinical experience has shown that the method can be used to restore the thumb and one of the three-phalangeal fingers in the presence of a recipient finger stump at the base of the main phalanx and within the distal third of the metacarpal in the absence of all three-phalangeal fingers. With several stumps of threephalangeal fingers on the donor hand, the question arises about using one of them as a donor. In our opinion, the most crippled finger (it is rare in frostbite) or the shortest stump should be considered for transplantation with use of precision technology that is in line with the data of other authors [13]. Our experience of using finger stumps to reconstruct another finger shows tha the loss of the stump does not affect the total function of the hand. A full-length finger can be transplanted if the function is significantly limited or absent, and there are prospects for the substantial improvement or restoration with the reconstruction of the thumb and the ulnar branch. This approach minimizes the cosmetic and functional donor defect that is in line with the results of other authors $[10,11]$. Transplantation of foot tissue complexes is reported to be associated with gait disorders in $23.7 \%$ of patients [2] and a donor defect of different severity $[5,18,19]$. Transplantation of a denervated finger of a paralyzed limb with joint contractures can hardly be considered [11]. Preservation of the finger vessels of the donor stump is vital and vascular examination of the fingers, both hands, forearms would be practical for identification of structural changes with the Allen test being advisable for the donor and recipient hands. In this regard, the method of ultrasonic Dopplerography is highly informative [20]. The operation can be performed in the presence of confined palmar arches on the donor and recipient hands. Fluid and anticoagulant therapy are recommended for 7-10 days due to the risk of vascular microanastomosis.

\section{CONCLUSION}

Reconstruction of the thumb or three-phalangeal finger by transplanting the stump of the finger of the opposite hand by microvascular anastomoses in patients with frostbite is an effective treatment method and be used according to indications considering the nature of defects, the level of amputation of the hand stump and vascular environment of the donor and recipient hands.

\section{REFERENCES}

1. Nakanishi A., Kawamura K., Omokawa S., Shimizu T., Iida A., Tanaka Y. Quality of life in patients with toe-to-hand transplantation. J. Plast. Surg. Hand Surg., 2018, vol. 52, no. 6, pp. 359-362. DOI: 101080/2000 656X20181520123.

2. Sosin M., Lin C.H., Steinberg J., Hammond E.R., Poysophon P., Iorio M.L., Patel K.M. Functional donor site morbidity after vascularized toe transfer procedure: a review of the literature and biomechanical consideration for surgical site selection. Ann. Plast. Surg., 2016, vol. 76, no. 6, pp. 735-742. DOI: 10.1097/SAP.0000000000000591.

3. Jones N.F., Hansen S.L., Bates S.J. Toe-to-hand transfers for congenital anomalies of the hand. Hand. Clin., 2007, vol. 23, no. 1, pp. 129-136. DOI: 10.1016/j.hcl.2007.02.006. 
4. Kvernmo H.D., Tsai T.M. Posttraumatic reconstruction of the hand - a retrospective review of 87 toe-to-hand transfers compared with an earlier report. J. Hand Surg. Am., 2011, vol. 36, no. 7, pp. 1176-1181. DOI: 10.1016/j.jhsa.2011.04.010.

5. Waljee J.F., Chung K.C. Toe-to-hand transfer: evolving indications and relevant outcomes. J. Hand Surg. Am., 2013, vol. 38, no. 7, pp. 1431-1434. DOI: 10.1016/j.jhsa.2013.03.020.

6. Tsai T.Y., Fries C.A., Hsiao J.C., Hsu C.C., Lin Y.T., Chen S.H., Lin C.H., Wei F.C., Lin C.H. Patient-reported outcome measures for toe-to-hand transfer: a prospective longitudinal study. Plast. Reconstr. Surg., 2019, vol. 143, no. 4, pp. 1122-1132. DOI: 10.1097/ PRS. 0000000000005422.

7. Sachs C., Lehnhardt M., Daigeler A., Goertz O. The triaging and treatment of cold-induced injuries. Dtsch. Arztebl. Int., 2015, vol. 112 , no. 44, pp. 741-747. DOI: 10. 3238/arztebl. 2015.0741.

8. Foucher G., Nagel D. Pinch reconstruction by hand to hand finger transfer associated with hallux transfer after a severe frostbite injury. J. Hand Surg. Br., 1999, vol. 24, no. 5, pp. 617-620. DOI: 10.1054/jhsb.1999.0225.

9. Brüser P., Larkin G. Contralateral transplantation of a finger for restoration of thumb function. J. Hand Surg. Br., 1997, vol. 22, no. 3, pp. 336-340. DOI: 10.1016/s0266-76819780398-1.

10.Jabłecki J., Kaczmarzyk J., Kaczmarzyk L. Transfer palca reki przeciwstawnej na reke bezpalcowa--ilustracja przypadku [Finger transfer from contralateral hand on metacarpal hand - case illustration]. Chir. Narzadow Ruchu Ortop. Pol., 2009, vol. 74, no. 5, pp. 305-308. (in Polish)

11.Giot J.P., Paek L.S., Mercier-Couture G., Coeugniet E., Bou-Merhi J.S., Harris P.G., Danino M.A. Free transfer of a paralyzed contralateral little finger for total thumb reconstruction in an electrical burn patient: a case report and literature review. J. Burn Care Res., 2016, vol. 37, no. 6, pp. e595-e600. DOI:10.1097/BCR.0000000000000333.

12.Parin B.V. Rekonstruktsiia paltsev ruki [Reconstruction of the hand fingers]. M., Medgiz, 1944, 130 p. (in Russian)

13.Sasaki K., Nozaki M., Takeuchi M. Thumb reconstruction, after Marjolin's ulcer resection by microvascular transfer of a burncontracted little finger: a case report of spare part surgery. Br. J. Plast. Surg., 1998, vol. 51, no. 2, pp. 126-127. DOI: 10.1054/ bjps.1997.0095.

14.Zhao J., Tien H.Y., Abdullah S., Zhang Z. Aesthetic refinements in second toe-to-thumb transfer surgery. Plast. Reconstr. Surg., 2010, vol. 126, no. 6, pp. 2052-2059. DOI: 10. 1097/PRS.0b013e3181f44994.

15.Henry S.L., Wei F.C. Thumb reconstruction with toe transfer. J. Hand Microsurg., 2010, vol. 2, no. 2, pp. 72-78. DOI: 101007/ s12593-010-0017-4.

16.Kumta S.M. Unfavourable results in thumb reconstruction. Indian J. Plast. Surg., 2013, vol. 46, no. 2, pp. 294-302. DOI: 10.4103/09700358.118608.

17.Lin Y.T., Loh C.Y.Y., Lien S.H., Lin C.H., Wei F.C. Simultaneous Stiles-Bunnell tendon transfer enhances intrinsic function of second-toe transplantations. Plast. Reconstr. Surg., 2017, vol. 140, no. 6, pp. 1229-1234. DOI: 101097/PRS.0000000000003861.

18.Yang C., Hsieh Y.H., Chen H.H., Tsai C.H., Lin Y.T. Gait improvement and ulcer prevention with second-to-great toe transposition at the donor site: a case report. Ann. Plast. Surg., 2020, vol. 84, no. 1S Suppl.1, pp. S128-S131. DOI: 10.1097/SAP.0000000000002200.

19.Le V.D., Nguyen V.T., Nguyen V.T. Use of single-staged transmetatarsal trimmed great toe transfer for reconstruction of a thumb amputation at the carpometacarpal joint. J. Plast. Reconstr. Aesthet. Surg., 2021, vol. 74, no. 5, pp. 1004-1012. DOI: 10.1016/j. bjps.2020.10.071.

20.Dolganova T.I., Shabalin D.A., Shikhaleva N.G. Ultrazvukovaia dopplerografiia paltsevykh arterii pri udlinenii kultei falang paltsev mini-fiksatorami [Ultrasound Doppler ultrasonography of digital arteries with lengthening of phalangeal stumps with mini fixators]. Travmatologiia i Ortopediia Rossii. 2022. Vol. 28, no. 1. P. XXX-XXX.

The article was submitted 15.01.2021; approved after reviewing 24.03.2021; accepted for publication 23.12.2021.

\section{Information about the authors:}

1. Nikolai Mikhailovich Aleksandrov - Doctor of Medical Sciences, aleksandrov-chetai@rambler.ru;

2. Ivan Denisovich Veshaev - M.D.;

3. Dmitry Alekseevich Kuptsov - M.D.

Funding The authors received no financial support for the research and/or authorship of this article. 